\title{
Role of rhinomanometry in the prediction of therapeutic positive airway pressure for obstructive sleep apnea
}

\author{
Yen-Bin Hsu ${ }^{1,2}$, Stanley Yung-Chuan Liư ${ }^{3}$, Ming-Ying Lan ${ }^{1,2}$, Yun-Chen Huang ${ }^{4,5}$, I-Shiang Tzeng ${ }^{6}$ and \\ Ming-Chin $\operatorname{Lan}^{4,5^{*}}$ (D)
}

\begin{abstract}
Background: This study was conducted to evaluate the relationship between nasal resistance in different posture and optimal positive airway pressure (PAP) level. Other potential factors were also assessed for possible influence on PAP pressure.

Methods: Forty- three patients diagnosed with obstructive sleep apnea (OSA) were prospectively recruited in this study. Nasal resistance was assessed by active anterior rhinomanometry in a seated position and then in a supine position at pressures of 75, 150, and 300 pascal. The factors correlating with PAP pressure were analyzed, including nasal resistance and patients' clinical data.

Results: Univariate analysis revealed that PAP pressure was correlated to nasal resistance in the supine position at 75 and 150 pascal (SupineNR75 and SupineNR150) ( $P=0.019$ and $P=0.004$ in Spearman's correlation coefficient analysis), but not correlated to nasal resistance in the seated position at different pressures or in the supine position at 300 pascal. The multiple linear regression analysis revealed that both SupineNR150 and body mass index (BMI) significantly predicted PAP pressure ( $\beta=0.308, p=0.044 ; \beta=0.727, p=0.006)$. The final PAP pressure predictive model was: PAP pressure $=0.29 \mathrm{BMl}+2.65$ SupineNR150 +2.11 .

Conclusions: Nasal resistance in the supine position measured at 150 pascal may provide valuable information regarding optimal PAP pressure. Rhinomanometry should be included in the treatment algorithm of OSA patients when PAP therapy is considered.
\end{abstract}

Keywords: Nasal resistance, Obstructive sleep apnea, Positive airway pressure, CPAP, Rhinomanometry

\section{Backgrounds}

Positive airway pressure (PAP) device is generally considered the first-line treatment for moderate to severe obstructive sleep apnea (OSA) [1]. PAP device serves as a "pneumatic splint" to overcome upper airway collapsibility via positive pressure from a tightly sealed

\footnotetext{
* Correspondence: lanmingchin@gmail.com

${ }^{4}$ Department of Otolaryngology-Head \& Neck Surgery, Taipei Tzu Chi Hospital, Buddhist Tzu Chi Medical Foundation, New Taipei City, Taiwan

${ }^{5}$ School of Medicine, Tzu Chi University, Hualien, Taiwan

Full list of author information is available at the end of the article
}

nasal or oral mask. Randomized controlled trials have shown benefits of PAP device, which include improvements in daytime sleepiness, cognitive performance, blood pressure, and overall quality of life [2-6]. However, the efficacy of PAP therapy is limited by poor acceptance and compliance. It has been reported that rates of compliance approximate 50\% [7]. An often cited reason for PAP intolerance refers to nasal problems, which accounts for $30-50 \%$ of cases [8]. These side effects include nasal congestion, postnasal drip, crusting, mucosal dryness, and recurrent sinusitis $[9,10]$. 
Optimal PAP titration is an important issue. There are several methods to determine optimal PAP titration: (1) conventional polysomnography (PSG) in the sleep laboratory, which is time consuming and expensive; (2) split night PSG, which combines diagnostic PSG in the first half of night and therapeutic PSG in the second half of night; (3) automatic positive airway pressure (APAP), which determines optimal pressure level by individual device algorithms; and (4) mathematical formulas comprised of PSG parameters and anthropometric variables in prediction models [11].

The mathematical formulas which predict optimal pressure may reduce time and cost at the sleep lab during initial PAP titration studies. Previous studies have demonstrated that PSG parameters [apnea-hypopnea index (AHI), oxygen desaturation index (ODI) and mean oxygen saturation] and anthropometric variables [body mass index (BMI), neck circumference, Friedman's tongue position and hyoid-mental distance] were established variables in a mathematical model to predict PAP pressure $[12,13]$.

Although the nasal airway contributes more than 50\% of upper airway resistance, few studies have incorporated nasal resistance as a potential predictor to evaluate optimal PAP pressure [11]. Currently, nasal resistance is thought to be a contributing factor for snoring and mild OSA, but does not play a major role in moderate to severe OSA $[14,15]$.

In this study, rhinomanometry was used to assess the degree of nasal resistance. Nasal resistance was measured in the seated and supine position at different pressures. The aim of this study was to investigate the influence of nasal resistance in different positions on optimal PAP pressure.

\section{Methods}

\section{Subjects and procedures}

From January 2018 to March 2019, 43 subjects aged between 20 to 70 years were recruited prospectively. All subjects were diagnosed with OSA if they had AHI $\geq 5$ episodes per hour of sleep and OSA-related symptoms. Exclusion criteria included smoking, previous nasal surgery, and diagnosis of nasal polyp or tumor. Subjects were instructed not to use any oral or topical nasal medications one week before rhinomanometric measurement. Subjects underwent comprehensive examination, including anterior rhinoscopy, nasopharyngoscopy, active anterior rhinomanometry, polysomnography and continuous positive airway pressure (CPAP) titration at the sleep lab. Modified Mallampati score and Epworth Sleepiness Scale were obtained. This study was approved by the Taipei Tzu Chi Hospital Institutional Review Board (no. 05-X06-014).

\section{Rhinomanometry}

Subjects were instructed to rest in a quiet room with temperature control to maintain a relaxed state for 5 min [16]. Nasal resistance was assessed by active anterior rhinomanometry. All rhinomanometric measurements were performed using the NR 6-rhinomanomer (GM Instruments Ltd., Glasgow, UK) at pressures of 75, 150, and 300 pascal. The assessment was first in the seated position and then in the supine position. All the tests were finished in $25 \mathrm{~min}$ to avoid the possible effects from nasal cycle.

\section{Polysomnography}

Standard PSG was performed in all subjects by trained sleep technicians. Electroencephalography, electrooculography, electrocardiography and electromyography for submentalis and tibialis anterior muscle were monitored continuously. Oronasal airflow was measured by thermistor. Thoraco-abdominal movements were recorded by piezoelectric bands. Apnea events were classified into obstructive apnea, central apnea and mixed apnea. An obstructive apnea event was defined as an absence or greater than $90 \%$ reduction in baseline airflow for at least $10 \mathrm{~s}$ with continued respiratory effort detected by the chest and abdomen movement channels. A hypopnea event was defined as greater than 30\% reduction in the baseline airflow for at least $10 \mathrm{~s}$ and associated with $3 \%$ oxygen desaturation or an electroencephalogram arousal. AHI represented the total number of apnea and hypopnea events per hour of sleep.

\section{Optimal PAP titration}

All subjects underwent nasal CPAP titration. All procedures were performed based on the criteria described in the American Academy of Sleep Medicine (AASM) clinical guidelines. The nasal masks were fitted by trained sleep technicians during the period of calibration. An optimal titration indicates that AHI is less than 5 events per hour for at least a 15-min duration, and supine REM sleep at the selected pressure is not continually interrupted by spontaneous arousal or awakenings [17].

\section{Statistical analysis}

All statistical analysis was carried out using SPSS version 20.0 (IBM Corp., Armonk, NY). Continuous data was expressed as mean \pm standard deviation, while categorical data was expressed as numbers and percentages. Spearman's rank correlation coefficient was utilized to evaluate the correlation between PAP pressure and nasal resistance in the seated position and in the supine position at different pressures. A linear regression model was developed to identify significant contributors to optimal PAP pressure. Differences between groups were compared using Mann-Whitney U 
test for nonparametric continuous data. $\mathrm{P}$ value less than 0.05 was considered statistically significant.

\section{Results}

Of the 43 OSA subjects with complete records, there were 32 males $(74.4 \%)$ and 11 females (25.6\%). The mean age was 48.05 years (range, $25-68$ years), and the mean BMI was $27.81 \mathrm{~kg} / \mathrm{m}^{2}$ (range, $19.40-41.00 \mathrm{~kg} / \mathrm{m}^{2}$ ). Demographic data is shown in Table 1. AHI ranged from 6.90 to 93.20 events per hour, with a mean of 43.04 events per hour. ODI ranged from 4.90 to 89.90 events per hour, with a mean of 36.43 events per hour. PAP pressure ranged from 6 to $12 \mathrm{~cm}$ of water pressure (cwp), with a mean of $8.42 \pm 1.8 \mathrm{cwp}$.

Spearman's correlation coefficient analysis showed strong positive correlations between PAP pressure and BMI $(\mathrm{r}=0.73, P<0.001)$ and AHI $(\mathrm{r}=0.62, P<0.001)$ (Table 2). Moderate positive correlation was noted between PAP pressure and nasal resistance in the supine position at 150 pascal (SupineNR150; $r=0.49, P=$ $0.004)$ and age $(\mathrm{r}=-0.458, P=0.002)$ and neck circumference $(\mathrm{r}=0.553, P<0.001)$. Weak positive agreement was noted between PAP pressure and nasal resistance in the supine position at 75 pascal (SupineNR75; $\mathrm{r}=0.37, P$ $=0.019$ ). No significant correlations were found between PAP pressure and nasal resistance in the seated position at 75,150 and 300 pascal (SeatedNR75, SeatedNR150, and SeatedNR300).

A multiple linear regression analysis was used to test if PSG parameters, anthropometric factors and nasal resistance could predict optimal PAP pressure. SupineNR150 significantly predicted optimal PAP pressure ( $\beta=0.308, p=0.044)$, as did BMI $(\beta=0.727, p=0.006)$. The results of the regression indicated two predictors accounting for $70.5 \%$ of the variance $[\mathrm{R} 2=0.705, \mathrm{~F}(8$, 23) $=6.86, p<0.001]$.

Table 1 Patient characteristics

\begin{tabular}{lll}
\hline Variables & Median & Range \\
\hline Males $(\mathrm{n}, \%)$ & $32(74.4 \%)$ & - \\
Females $(\mathrm{n}, \%)$ & $11(25.6 \%)$ & - \\
Age, years & 49.00 & $25-68$ \\
BMI, kg/m ${ }^{2}$ & 26.80 & $19.40-41.00$ \\
ODI, events/hr & 34.40 & $4.90-89.90$ \\
AHI, events/hr & 40.50 & $6.90-93.20$ \\
PAP pressure & 8 & $6-12$ \\
Neck circumference, cm & 39 & $30-46$ \\
ESS & 10 & $2-22$ \\
Tonsil size & 1 & $0-2$ \\
MMS & 3 & $2-4$ \\
\hline
\end{tabular}

$B M I$ body mass index, ODI oxygen desaturation index, $A H I$ apnea-hypopnea index, PAP positive airway pressure, ESS Epworth Sleepiness Scale, MMS Modified Mallampati score
Table 2 Spearman's correlation between positive airway pressure level and collected variables

\begin{tabular}{llc}
\hline Variables & Coefficient & $P$ Value \\
\hline Age, years & -0.46 & 0.002 \\
BMI, kg/m² & 0.73 & $<0.001$ \\
AHI, events/hr & 0.62 & $<0.001$ \\
ESS & 0.15 & 0.342 \\
Neck circumference, cm & 0.55 & $<0.001$ \\
Tonsil size & 0.28 & 0.070 \\
MMS & 0.19 & 0.239 \\
SeatedNR75 & 0.06 & 0.711 \\
SeatedNR150 & -0.19 & 0.265 \\
SeatedNR300 & -0.07 & 0.733 \\
SupineNR75 & 0.37 & 0.019 \\
SupineNR150 & 0.49 & 0.004 \\
SupineNR300 & 0.42 & 0.057 \\
\hline BMI body
\end{tabular}

$B M I$ body mass index, $A H I$ apnea-hypopnea index, ESS Epworth Sleepiness Scale, MMS Modified Mallampati score, SeatedNR75, SeatedNR150, SeatedNR300 nasal resistance in the seated position at 75, 150 and 300 pascal, SupineNR75, SupineNR150, SupineNR300 nasal resistance in the supine position at 75, 150 and 300 pascal

The final PAP pressure predictive model was:

PAP pressure $=0.29 \mathrm{BMI}+2.65$ SupineNR $150+2.11$

This model indicated that optimal PAP pressure is significantly correlated with BMI and SupineNR150 (Table 3).

Subjects with PAP pressure greater than $8 \mathrm{cwp}$ were associated with a significantly higher SupineNR150 $(P=$ 0.018) compared to patients with PAP pressure less than or equal to $8 \mathrm{cwp}$. No significant associations were found between nasal resistance in the seated position at 75, 150 and 300 pascal (SeatedNR75, SeatedNR150,

Table 3 Multiple linear regression analysis to predict the potential variables for positive airway pressure level.

\begin{tabular}{|c|c|c|c|c|}
\hline Variables & B & $\beta$ & $95 \% \mathrm{Cl}$ & $P$ Value \\
\hline Sex & -0.74 & -0.20 & $-2.11-0.64$ & 0.280 \\
\hline Age, years & 0.002 & 0.01 & $-0.04-0.05$ & 0.927 \\
\hline $\mathrm{BMI}, \mathrm{kg} / \mathrm{m} 2$ & 0.29 & 0.73 & $0.09-0.48$ & 0.006 \\
\hline AHI, events/hr & 0.01 & 0.17 & $-0.01-0.03$ & 0.235 \\
\hline Neck circumference, cm & -0.06 & -0.11 & $-0.33-0.21$ & 0.639 \\
\hline Tonsil size & -0.23 & -0.05 & $-1.54-1.09$ & 0.726 \\
\hline MMS & 0.11 & 0.04 & $-0.68-0.90$ & 0.774 \\
\hline SupineNR75 & 1.52 & 0.19 & $-0.49-3.52$ & 0.133 \\
\hline SupineNR150 & 2.65 & 0.31 & $0.003-5.29$ & 0.044 \\
\hline
\end{tabular}

$B M I$ body mass index, $A H I$ apnea-hypopnea index, MMS Modified Mallampati score, SupineNR75, SupineNR150 nasal resistance in the supine position at 75 and 150 pascal 
SeatedNR300) and different degrees of PAP pressure (PAP pressure $\leq 8$ or $>8 \mathrm{cwp}$ ). (Table 4 and Fig. 1 ).

\section{Discussion}

Conventional PSG with manual titration of optimal PAP pressure is considered the gold standard method for starting PAP therapy. However, it can be costly and time-consuming. Researchers have long been exploring for reliable mathematic models to predict optimal therapeutic PAP pressure settings. Several potential predictors to formulate mathematic models, which include BMI, neck circumference, AHI, ODI or mean oxygen saturation, were previously explored $[12,13]$. However, nasal resistance has rarely been examined as a potential variable to estimate optimal therapeutic PAP pressure setting. This study aimed to explore the relationship between nasal resistance in different posture and optimal PAP pressure.

Previous studies have reported that subjective perception of nasal obstruction was poorly correlated with actual nasal resistance [18-20]. Active anterior rhinomanometry, which assesses nasal pressure and airflow during respiration, is able to determine the nasal resistance objectively. It is good for assessing the presence and severity of obstruction [21]. Although there are some shortcomings of this technique, including incapability to detect obstructive area precisely and the need of specialized equipment and a well-trained operator, active anterior rhinomanometry is still the most commonly used method and also considered the gold standard for evaluation of nasal resistance [22, 23].

Table 4 Associations between nasal resistance and positive airway pressure level.

\begin{tabular}{llll}
\hline Nasal Resistance & & Mean \pm SD & P Value \\
\hline SeatedNR75 & PAP pressure $\leq 8$ & $0.155 \pm 0.459$ & 0.970 \\
& PAP pressure $>8$ & $0.162 \pm 0.023$ & \\
SeatedNR150 & PAP pressure $\leq 8$ & $0.215 \pm 0.061$ & 0.165 \\
& PAP pressure $>8$ & $0.217 \pm 0.028$ & \\
SeatedNR300 & PAP pressure $\leq 8$ & $0.307 \pm 0.084$ & 0.467 \\
& PAP pressure $>8$ & $0.295 \pm 0.036$ & \\
SupineNR75 & PAP pressure $\leq 8$ & $0.170 \pm 0.074$ & 0.060 \\
& PAP pressure $>8$ & $0.211 \pm 0.043$ & \\
SupineNR150 & PAP pressure $\leq 8$ & $0.224 \pm 0.091$ & $0.018^{\text {a }}$ \\
& PAP pressure $>8$ & $0.281 \pm 0.055$ & \\
SupineNR300 & PAP pressure $\leq 8$ & $0.313 \pm 0.116$ & 0.140 \\
& PAP pressure $>8$ & $0.379 \pm 0.075$ & \\
\hline
\end{tabular}

PAP positive airway pressure, SeatedNR75, SeatedNR150, SeatedNR300 nasal resistance in the seated position at 75, 150 and 300 pascal, SupineNR75, SupineNR150, SupineNR300 nasal resistance in the supine position at 75, 150 and 300 pascal

${ }^{a}$ Indicates statistical significance

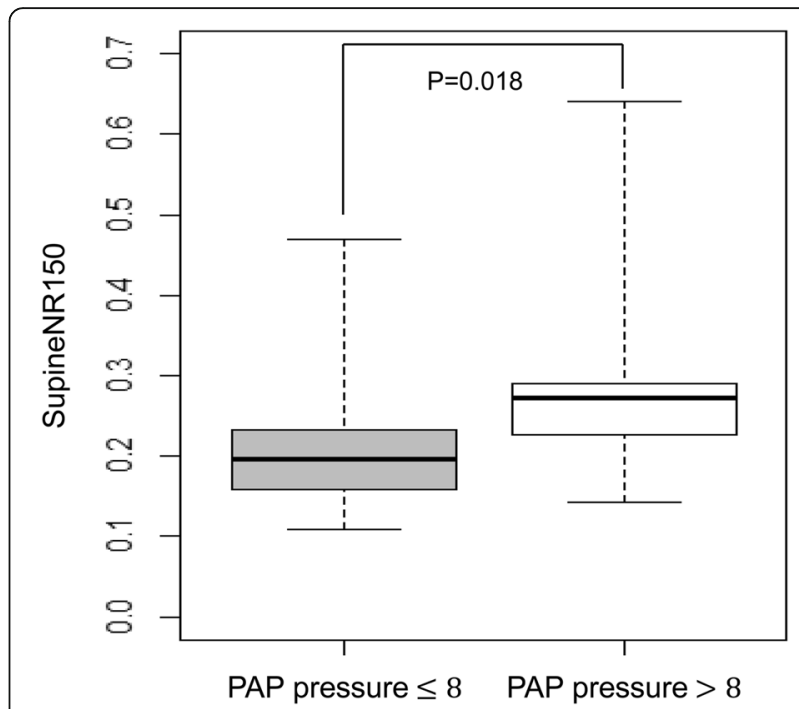

Fig. 1 Patients with PAP pressure $>8$ were associated with a significantly higher SupineNR150 compared to patients with PAP pressure $\leq 8$. PAP, positive airway pressure; SupineNR 150, nasal resistance in the supine position at 150 pascal

In this study, multiple linear regression analysis indicated that BMI and SupineNR150 were contributing factors to PAP pressure. The influence of nasal resistance was less than that of BMI $(\beta=0.308, p=0.044$ vs $\beta=$ $0.727, p=0.006$ ). Hueto et al. had previously shown that BMI and nasal resistance in the supine position after vasoconstriction at rhinomanometry pressure of 150 pascal were significant predictors of pressure settings [11]. However, the nasal resistance was measured after vasoconstriction in their study, whereas we measured nasal resistance without the use of a decongestant. Topical nasal decongestants raise vasoconstrictor tone which in turn increases nasal patency and reduces nasal resistance. Our study design approximates a more native physiologic environment.

Although nasal resistance is not considered a significant contributor in patients with moderate to severe OSA, it does play an important role in CPAP treatment. Nakata et al. have indicated that nasal resistance was higher in nasal CPAP failure patients compared to CPAP compliant patients, and the PAP titration level decreased significantly after nasal surgery [24]. Camacho et al. concluded in the systematic review and meta-analysis that nasal surgery not only decreased therapeutic CPAP pressure but also increased CPAP compliance [25]. Sugiura et al. have shown that nasal resistance and $\mathrm{AHI}$ were statistically significant variables to predict acceptance of CPAP, and a higher nasal resistance might result in CPAP non-acceptance [26]. Morris et al. have found that nasal cross-sectional area at the head of inferior turbinate differed significantly between CPAP-tolerant and CPAP-intolerant patients, supporting a major role of nasal resistance in CPAP 
therapies [18]. Powell et al. have demonstrated that radiofrequency reduction of turbinate hypertrophy significantly increased adherence to CPAP [27].

Of interest to our study design which included assessment of nasal resistance in the supine position, Tarrega et al. previously reported that nasal resistance assessed by active anterior rhinomanometry correlated poorly with PAP treatment levels [28]. Nonetheless, they measured nasal resistance only in the seated position, which could not represent the natural sleep position. The measurements might not accurately reflect genuine nasal condition during sleep. In this study, we also found no significant correlations between PAP pressure and nasal resistance in the seated position at different pressures. However, we noted that nasal resistance in the supine position measured at 150 pascal was a significant predictor of therapeutic PAP pressure.

There are several limitations in this study. First, the study population is mainly comprised of patients with moderate to severe OSA because CPAP is considered as first-line therapy for patients with moderate to severe OSA. Therefore, the results may not fully represent the general OSA population. Second, the influence of nasal cycle may result in side to side variation of nasal resistance over a period of hours. Nevertheless, the total nasal resistance remains rather stable despite side to side fluctuation of nasal resistance. In this study, we measured total nasal resistance to prevent the possible effect of nasal cycle. Third, the results of rhinomanometry measurements might be partially influenced by adaptations to environmental changes. The difference of nasal resistance between the seated and supine positions may result from better acclimation to the room atmosphere. Therefore, all subjects in this study were instructed to rest for at least $5 \mathrm{~min}$ in the examination room before being assessed by active anterior rhinomanometry to minimize the effect of acclimation. Finally, the study population is relatively small. Larger studies may be warranted to confirm the role of rhinomanometry in the prediction of therapeutic PAP pressure for OSA.

\section{Conclusions}

Nasal resistance in the supine position at 150 pascal is a significant predictor for optimal PAP pressure. An objective assessment of nasal obstruction by rhinomanometry is warranted in OSA patients when considering CPAP treatment. Nasal resistance in the supine position may provide valuable information regarding optimal PAP pressure and further need for nasal surgery.

\section{Abbreviations}

AASM: American Academy of Sleep Medicine; AHI: Apnea-hypopnea index; APAP: Automatic positive airway pressure; BMI: Body mass index; CPAP. Continuous positive airway pressure; $\mathrm{Cwp}$ : $\mathrm{Cm}$ of water pressure:

ODI: Oxygen desaturation index; OSA: Obstructive sleep apnea; PAP: Positive airway pressure; PSG: Polysomnography; SeatedNR75, SeatedNR150, SeatedNR300: Nasal resistance in the seated position at 75, 150 and 300 pascal; SupineNR75, SupineNR150, SupineNR300: Nasal resistance in the supine position at 75, 150 and 300 pascal

\section{Acknowledgements}

This study was funded by Taipei Tzu Chi Hospital, Buddhist Tzu Chi Medical Foundation (grant number TCRD-TPE-108-26).

\section{Authors' contributions}

$M C L$ and $Y B H$ conceived and designed the study, analyzed the data and drafted this manuscript. SYL, MYL, YCH and IST contributed to analysis of the data, and revising of the manuscript. All authors have read and approved the final manuscript.

\section{Funding}

This study was supported by grants from Taipei Tzu Chi Hospital, Buddhist Tzu Chi Medical Foundation (TCRD-TPE-108-26).

Availability of data and materials

All data generated or analyzed during the present study are included in this published article.

\section{Ethics approval and consent to participate}

The study protocol was approved by the local institutional review board (no. 05-X06-014) and all subjects gave signed informed consent.

\section{Consent for publication}

Not applicable.

\section{Competing interests}

The authors declare that they have no competing interests.

\section{Author details}

${ }^{1}$ Department of Otolaryngology-Head \& Neck Surgery, Taipei Veterans General Hospital, Taipei, Taiwan. ${ }^{2}$ School of Medicine, National Yang-Ming University, Taipei, Taiwan. ${ }^{3}$ Division of Sleep Surgery, Department of Otolaryngology, Stanford University School of Medicine, Stanford, CA, USA. ${ }^{4}$ Department of Otolaryngology-Head \& Neck Surgery, Taipei Tzu Chi Hospital, Buddhist Tzu Chi Medical Foundation, New Taipei City, Taiwan. ${ }^{5}$ School of Medicine, Tzu Chi University, Hualien, Taiwan. ${ }^{6}$ Department of Research, Taipei Tzu Chi Hospital, Buddhist Tzu Chi Medical Foundation, New Taipei City, Taiwan.

Received: 16 January 2020 Accepted: 3 May 2020

Published online: 13 May 2020

\section{References}

1. Cao MT, Sternbach JM, Guilleminault C. Continuous positive airway pressure therapy in obstuctive sleep apnea: benefits and alternatives. Expert Rev. Respir Med. 2017;11(4):259-72.

2. Thiel S, Haile SR, Peitzsch M, Schwarz El, Sievi NA, Kurth S, et al. Endocrine responses during CPAP withdrawal in obstructive sleep apnoea: data from two randomised controlled trials. Thorax. 2019.

3. Bouloukaki I, Mermigkis C, Tzanakis N, Giannadaki K, Mauroudi E, Moniaki V, et al. The role of compliance with PAP use on blood pressure in patients with obstructive sleep apnea: is longer use a key-factor? J Hum Hypertens. 2017;31(2):106-15

4. Campos-Rodriguez F, Queipo-Corona C, Carmona-Bernal C, Jurado-Gamez B, Cordero-Guevara J, Reyes-Nunez N, et al. Continuous Positive Airway Pressure Improves Quality of Life in Women with Obstructive Sleep Apnea. A Randomized Controlled Trial. Am J Respir Crit Care Med. 2016;194(10):1286-94.

5. Culebras A. Sleep apnea and stroke. Curr Neurol Neurosci Rep. 2015;15(1):503.

6. Weaver TE, Mancini C, Maislin G, Cater J, Staley B, Landis JR, et al. Continuous positive airway pressure treatment of sleepy patients with milder obstructive sleep apnea: results of the CPAP Apnea Trial North American Program (CATNAP) randomized clinical trial. Am J Respir Crit Care Med. 2012;186(7):677-83.

7. Riachy M, Najem S, Iskandar M, Choucair J, Ibrahim I, Juvelikian G. Factors predicting CPAP adherence in obstructive sleep apnea syndrome. Sleep Breath. 2017;21(2):295-302. 
8. Mickelson SA. Nasal Surgery for Obstructive Sleep Apnea Syndrome. Otolaryngol Clin North Am. 2016;49(6):1373-81.

9. Balsalobre L, Pezato R, Gasparini H, Haddad F, Gregorio LC, Fujita RR. Acute impact of continuous positive airway pressure on nasal patency. Int Forum Allergy Rhinol. 2017;7(7):712-7.

10. Aguilar F, Cisternas A, Montserrat JM, Avila M, Torres-Lopez M, Iranzo A, et al. Effect of Nasal Continuous Positive Pressure on the Nostrils of Patients with Sleep Apnea Syndrome and no Previous Nasal Pathology. Predictive Factors for Compliance. Arch Bronconeumol. 2016;52(10):519-26.

11. Hueto J, Santaolalla F, Sanchez-Del-Rey A, Martinez-Ibarguen A. Usefulness of rhinomanometry in the identification and treatment of patients with obstructive sleep apnoea: an algorithm for predicting the relationship between nasal resistance and continuous positive airway pressure. a retrospective study. Clin Otolaryngol. 2016;41(6):750-7.

12. Camacho M, Riaz M, Tahoori A, Certal V, Kushida CA. Mathematical Equations to Predict Positive Airway Pressures for Obstructive Sleep Apnea: A Systematic Review. Sleep Disord. 2015;2015:293868.

13. Lai CC, Friedman M, Lin HC, Wang PC, Hwang MS, Hsu CM, et al. Clinical predictors of effective continuous positive airway pressure in patients with obstructive sleep apnea/hypopnea syndrome. Laryngoscope. 2015;125(8):1983-7.

14. Georgalas C. The role of the nose in snoring and obstructive sleep apnoea: an update. Eur Arch Otorhinolaryngol. 2011;268(9):1365-73.

15. Sharma S, Wormald JCR, Fishman JM, Andrews P, Kotecha BT. Rhinological interventions for obstructive sleep apnoea - a systematic review and descriptive meta-analysis. J Laryngol Otol. 2019;133(3):168-76.

16. Kahana-Zweig R, Geva-Sagiv M, Weissbrod A, Secundo L, Soroker N, Sobel N. Measuring and Characterizing the Human Nasal Cycle. PLoS One. 2016; 11(10):e0162918.

17. Kushida CA, Chediak A, Berry RB, Brown LK, Gozal D, Iber C, et al. Clinical guidelines for the manual titration of positive airway pressure in patients with obstructive sleep apnea. J Clin Sleep Med. 2008;4(2):157-71.

18. Morris LG, Setlur J, Burschtin OE, Steward DL, Jacobs JB, Lee KC. Acoustic rhinometry predicts tolerance of nasal continuous positive airway pressure: a pilot study. Am J Rhinol. 2006;20(2):133-7.

19. Zhao K, Blacker K, Luo Y, Bryant B, Jiang J. Perceiving nasal patency through mucosal cooling rather than air temperature or nasal resistance. PLoS One. 2011;6(10):e24618.

20. Andre RF, Vuyk HD, Ahmed A, Graamans K, Nolst Trenite GJ. Correlation between subjective and objective evaluation of the nasal airway. A systematic review of the highest level of evidence. Clin Otolaryngol. 2009;34(6):518-25.

21. van Spronsen E, Ingels KJ, Jansen AH, Graamans K, Fokkens WJ. Evidencebased recommendations regarding the differential diagnosis and assessment of nasal congestion: using the new GRADE system. Allergy. 2008;63(7):820-33.

22. Spataro E, Most SP. Measuring Nasal Obstruction Outcomes. Otolaryngol Clin North Am. 2018:51(5):883-95.

23. Clement PA, Halewyck S, Gordts F, Michel O. Critical evaluation of different objective techniques of nasal airway assessment: a clinical review. Eur Arch Otorhinolaryngol. 2014;271(10):2617-25.

24. Nakata S, Noda A, Yagi H, Yanagi E, Mimura T, Okada T, et al. Nasal resistance for determinant factor of nasal surgery in CPAP failure patients with obstructive sleep apnea syndrome. Rhinology. 2005;43(4):296-9.

25. Camacho M, Riaz M, Capasso R, Ruoff CM, Guilleminault C, Kushida CA, et al. The effect of nasal surgery on continuous positive airway pressure device use and therapeutic treatment pressures: a systematic review and metaanalysis. Sleep. 2015;38(2):279-86.

26. Sugiura T, Noda A, Nakata S, Yasuda Y, Soga T, Miyata S, et al. Influence of nasal resistance on initial acceptance of continuous positive airway pressure in treatment for obstructive sleep apnea syndrome. Respiration. 2007;74(1):56-60.

27. Powell NB, Zonato Al, Weaver EM, Li K, Troell R, Riley RW, et al. Radiofrequency treatment of turbinate hypertrophy in subjects using continuous positive airway pressure: a randomized, double-blind, placebocontrolled clinical pilot trial. Laryngoscope. 2001;111(10):1783-90.

28. Tarrega J, Mayos M, Montserrat JR, Fabra JM, Morante F, Caliz A, et al. Nasal resistance and continuous positive airway pressure treatment for sleep apnea/hypopnea syndrome. Arch Bronconeumol. 2003;39(3):106-10.

\section{Publisher's Note}

Springer Nature remains neutral with regard to jurisdictional claims in published maps and institutional affiliations.

Ready to submit your research? Choose BMC and benefit from:

- fast, convenient online submission

- thorough peer review by experienced researchers in your field

- rapid publication on acceptance

- support for research data, including large and complex data types

- gold Open Access which fosters wider collaboration and increased citations

- maximum visibility for your research: over $100 \mathrm{M}$ website views per year

At BMC, research is always in progress.

Learn more biomedcentral.com/submissions 